\title{
On the Existence of Linear Pfaff Systems with Lower Characteristic Sets of Positive Lebesgue $m$-Measure
}

\author{
N. A. Izobov, S. G. Krasovskii, and A. S. Platonov \\ Institute of Mathematics, National Academy of Sciences, Minsk, Belarus \\ Mogilev State University, Mogilev, Belarus
}

Received January 8, 2009

\begin{abstract}
We prove the existence of an $n$-dimensional completely integrable Pfaff system with multidimensional time of dimension $m \geq 2$, with bounded infinitely differentiable coefficients, and with the set of lower characteristic vectors of its solutions having positive Lebesgue $m$ measure.
\end{abstract}

DOI: $10.1134 /$ S0012266109050036

We consider $n$-dimensional linear Pfaff systems

$$
\frac{\partial x}{\partial t_{i}}=A_{i}(t) x, \quad x \in \mathbb{R}^{n}, \quad n \geq 2, \quad i=1, \ldots, m, \quad t=\left(t_{1}, \ldots, t_{m}\right) \in \mathbb{R}_{+}^{m}, \quad m \geq 2, \quad\left(1_{m n}\right)
$$

with continuously differentiable coefficient matrices satisfying the complete integrability condition [1, pp. 14-24; 2, pp. 16-26]

$$
\frac{\partial A_{i}(t)}{\partial t_{j}}+A_{i}(t) A_{j}(t)=A_{j}(t) A_{i}(t)+\frac{\partial A_{j}(t)}{\partial t_{i}}, \quad i, j=1, \ldots, m, \quad t \in \mathbb{R}_{+}^{m},
$$

in $\mathbb{R}_{+}^{m}=\left\{t \in \mathbb{R}^{m}: t \geq 0\right\}$.

Let $\lambda[x] \in \mathbb{R}^{m}$ and $p[x] \in \mathbb{R}^{m}$ be some characteristic [3] and lower characteristic [3] vectors of a nontrivial solution $x: \mathbb{R}_{+}^{m} \rightarrow \mathbb{R}^{n} \backslash\{0\}$ of system $\left(1_{m n}\right)$; these vectors are $m$-dimensional analogs of the corresponding characteristic Lyapunov exponent and lower Perron exponent. In addition, the first of them - the characteristic vector $\lambda[x]$ - is determined by the relations

$$
\begin{aligned}
L_{x}(\lambda[x]) & \equiv \varlimsup_{t \rightarrow \infty} \frac{\ln \|x(t)\|-(\lambda[x], t)}{\|t\|}=0, \quad L_{x}\left(\lambda[x]-\varepsilon e_{i}\right)>0 \quad \forall \varepsilon>0, \\
e_{i} & =\underbrace{0, \ldots, 0,1}_{i}, 0, \ldots, 0) \in \mathbb{R}_{+}^{m}, \quad i=1, \ldots, m,
\end{aligned}
$$

and the second - the lower characteristic vector $p[x]$ — is determined by the conditions

$$
\begin{aligned}
l_{x}(p[x]) & \equiv \varliminf_{t \rightarrow \infty} \frac{\ln \|x(t)\|-(p[x], t)}{\|t\|}=0, \\
l_{x}\left(p[x]+\varepsilon e_{i}\right) & <0 \quad \forall \varepsilon>0, \quad i=1, \ldots, m .
\end{aligned}
$$

The sets $\Lambda_{x}=\bigcup \lambda[x]$ and $P_{x}=\bigcup p[x]$ are referred to as the characteristic set [3] and the lower characteristic set [4], respectively, of the nontrivial solution $x(t)$ of system $\left(1_{m n}\right)$; they are bounded closed convex [2] and concave [4] curves, respectively, in the case of two-dimensional time $t \in \mathbb{R}^{2}$.

It is known that the set of characteristic exponents (e.g., see [5, p. 15]) of an $n$-dimensional ordinary differential system consists of at most $n$ distinct numbers; i.e., such a system has at most $n$ nontrivial solutions with pairwise distinct Lyapunov exponents. There is no counterpart of the 
previous result for the analog of the Lyapunov characteristic exponent of a nontrivial solution of an ordinary differential system, that is, for the characteristic set $\Lambda_{x}$ of a nontrivial solution $x: \mathbb{R}_{+}^{m} \rightarrow \mathbb{R}^{n}$ of a linear completely integrable Pfaff system $\left(1_{m n}\right)$. Namely, it was proved in [6] that there exists an $n$-dimensional system $\left(1_{2 n}\right)$ with two-dimensional time $t \in \mathbb{R}_{+}^{2}$ that has a countable set $\left\{x_{1}(t), \ldots, x_{k}(t), \ldots\right\}$ of nontrivial solutions $x_{k}: \mathbb{R}_{+}^{2} \rightarrow \mathbb{R}^{n} \backslash\{0\}$ with pairwise distinct characteristic sets $\Lambda_{x_{k}}\left(\neq \Lambda_{x_{j}}\right.$ for arbitrary $\left.k \neq j\right)$. In addition, it was proved in [7] that each completely integrable Pfaff system $\left(1_{22}\right)$ has at most countably many solutions with pairwise distinct characteristic sets. Therefore, the entire characteristic set $\Lambda\left(A_{1}, A_{2}\right)=\bigcup_{x \neq 0} \Lambda_{x}$ of system $\left(1_{22}\right)$ has zero plane Lebesgue measure.

The situation is different for the Perron lower exponent and its analog, the lower characteristic set. In the case of ordinary differential systems $\left(1_{1 n}\right)$, there exist systems whose set of Perron lower exponents has positive Lebesgue measure [8]. The existence of $n$-dimensional completely integrable Pfaff linear systems $\left(1_{2 n}\right)$ with two-dimensional time and $\left(1_{3 n}\right)$ with three-dimensional time and lower characteristic sets $\Pi\left(A_{1}, A_{2}\right)$ of positive plane measure and $\Pi\left(A_{1}, A_{2}, A_{3}\right)$ of positive measure in the space $\mathbb{R}^{3}$ was proved in [4] and [9], respectively. We encounter the problem on the existence of systems $\left(1_{m n}\right)$ with a similar property and with time $t \in \mathbb{R}_{+}^{m}$ of arbitrary dimension $m$.

The present paper deals with the construction of an $n$-dimensional linear completely integrable system $\left(1_{m n}\right)$ with $m$-dimensional time $t \in \mathbb{R}_{+}^{m}, m \geq 2$, with bounded infinitely differentiable coefficients in $\mathbb{R}_{+}^{m}$, and with lower characteristic set

$$
\Pi\left(A_{1}, \ldots, A_{m}\right)=\bigcup_{x \neq 0} P_{x}
$$

of positive Lebesgue measure in the space $\mathbb{R}^{m}$. To this end, we first construct the lower characteristic set of the sum of special scalar exponentials.

Lemma. The lower characteristic set $P_{E}$ of the function

$$
E(t)=\sum_{i=1}^{m} e^{-a_{i} t_{i}}, \quad a_{i}=\text { const }, \quad m \geq 2, \quad t_{i} \geq 0,
$$

coincides with the set

$$
\Pi_{E} \equiv\left\{p \in \mathbb{R}_{-}^{m}: S(p) \equiv \sum_{i=1}^{m} \frac{p_{i}}{a_{i}}=-1\right\}
$$

Proof. First, let us prove the inequality $p \leq 0$ for any lower characteristic vector $p \in P_{E}$. Suppose the contrary: $p_{k}>0$ for some $k \in\{1, \ldots, m\}$. Then we have the inequalities

$$
l_{E}(p) \leq \varliminf_{\|t\|=t_{k} \rightarrow+\infty} \frac{\ln \left(m-1+e^{-a_{k} t_{k}}\right)-p_{k} t_{k}}{t_{k}}=-p_{k}<0
$$

contradicting the first condition $l_{E}(p)=0$ in the definition of a lower characteristic vector $p$.

Now let us prove the inclusion

$$
\Pi_{E} \subset P_{E}
$$

for the part of the plane $\sum_{i=1}^{m} p_{i} / a_{i}=-1$ in the $m$-dimensional quadrant $\mathbb{R}_{-}^{m}=\left\{p \in \mathbb{R}^{m}: p \leq 0\right\}$ of the space $\mathbb{R}^{m}$. Let

$$
p \in \Pi_{E}, \quad t \in \mathbb{R}_{+}^{m} \backslash\{0\}, \quad \text { and } \quad a_{k} t_{k}=\min _{i=1, \ldots, m}\left\{a_{i} t_{i}\right\}
$$

for some $k \in\{1, \ldots, m\}$. Then, by virtue of the already proved inequalities $-p_{i} \geq 0$, we have the estimates

$$
\ln E(t)-(p, t) \geq-a_{k} t_{k}-p_{k} t_{k}-\sum_{i \neq k}^{m} p_{i} \frac{a_{k}}{a_{i}} t_{k}=a_{k} t_{k}\left(-1-\sum_{i=1}^{m} \frac{p_{i}}{a_{i}}\right)=0,
$$


which imply that $l_{E}(p) \geq 0$. At the same time, on the ray

$$
T_{a}=\left\{t \in \mathbb{R}_{+}^{m} \backslash\{0\}: a_{i} t_{i}=a_{k} t_{k}, i=1, \ldots, m\right\}, \quad k \in\{1, \ldots, m\},
$$

we have the opposite estimate

$$
[\ln E(t)-(p, t)]_{t \in T_{a}} \leq \ln m-a_{k} t_{k}\left(-1-\sum_{i=1}^{m} \frac{p_{i}}{a_{i}}\right)=\ln m,
$$

which justifies the inequality $l_{E}(p) \leq 0$ and hence the desired relation $l_{E}(p)=0$.

For a vector $p \in \Pi_{E}$, let us prove the second necessary condition

$$
l_{E}\left(p+\varepsilon e_{k}\right)<0, \quad e_{k}=(\underbrace{0, \ldots, 0,1}_{k}, 0, \ldots, 0) \quad \forall \varepsilon>0 .
$$

Indeed, on the ray $T_{a}$, we have the estimate

$$
\left[\ln E(t)-\left(p+\varepsilon e_{k}, t\right)\right]_{t \in T_{a}} \leq \ln m-\varepsilon t_{k},
$$

which implies the desired inequality

$$
l_{E}\left(p+\varepsilon e_{k}\right) \leq-\frac{\varepsilon}{a_{k} r}, \quad r \equiv\left(\sum_{i=1}^{m} \frac{1}{a_{i}^{2}}\right)^{1 / 2}, \quad k=1, \ldots, m .
$$

The proof of the inclusion (4) is complete.

Now let us prove the coincidence of the sets $\Pi_{E}$ and $P_{E}$. To this end, we suppose the contrary: there exists a vector $p \in P_{E}$ such that $p \notin \Pi_{E}$. By virtue of the above-proved inequality $q \leq 0$, we have $p \leq 0$ for any lower characteristic vector $q \in P_{E}$; therefore, we have the following cases for the quantity

$$
S(p) \equiv \sum_{i=1}^{m} \frac{p_{i}}{a_{i}}
$$

1. $S(p) \in(-1,0]$.

2. $S(p)<-1$.

In case 1 , on the ray $T_{a}$, we have the relations

$$
\begin{aligned}
{\left.[\ln E(t)-(p, t)]\right|_{t \in T_{a}} } & =\ln m-a_{k} t_{k}(1+S(p)), \quad 1+S(p)>0, \quad t_{k} \geq 0 \\
\|t\| \|_{t \in T_{a}} & =a_{k} t_{k} r, \quad k \in\{1, \ldots, m\}, \quad 1+S(p)>0, \quad t_{k} \geq 0
\end{aligned}
$$

which imply that $l_{E}(p) \leq-(1+S(p)) r^{-1}<0$. This inequality shows that the case $S(p) \in(-1,0]$ is impossible.

Consider case 2. Obviously, there exists a vector $q \in \Pi_{E}$ for which the conditions $p \leq q \leq 0$ and $p \neq q$ are satisfied and the inequality $p_{l}<q_{l}$ holds for some $l \in\{1, \ldots, m\}$. Then an arbitrary vector $t \in \mathbb{R}_{+}^{m} \backslash\{0\}$ such that

$$
a_{k} t_{k}=\min _{i=1, \ldots, m}\left\{a_{i} t_{i}\right\}, \quad k \in\{1, \ldots, m\},
$$

satisfies the inequalities

$$
\begin{aligned}
\ln E(t)-(p, t)-\varepsilon t_{l} & =\ln E(t)-(q, t)+(q-p, t)-\varepsilon t_{l} \geq-a_{k} t_{k}\left(1+\sum_{i=1}^{m} \frac{q_{i}}{a_{i}}\right)+\left(q_{l}-p_{l}-\varepsilon\right) t_{l} \\
& =\left(q_{l}-p_{l}-\varepsilon\right) t_{l} \geq 0, \quad t \in \mathbb{R}_{+}^{m},
\end{aligned}
$$

for $\varepsilon \in\left(0, q_{l}-p_{l}\right)$. They imply the inequality $l_{E}\left(p+\varepsilon_{l}\right) \geq 0$ for all sufficiently small $\varepsilon>0$, whence we find that the vector $p$ is not a lower characteristic vector of the function $E: \mathbb{R}_{+}^{m} \rightarrow \mathbb{R}_{+} \backslash\{0\}$; 
therefore, the second case $S(p)<-1$ is impossible. We have thereby shown that each vector $p \in P_{E}$ belongs to the set $\Pi_{E}$. This, together with the above-proved inclusion (4), implies that $P_{E}=\Pi_{E}$. The proof of the lemma is complete.

The following assertion establishes the existence of the desired system.

Theorem. For arbitrary positive integers $m \geq 2$ and $n \geq 2$, for real numbers $\alpha_{1} \leq \alpha_{2} \leq 0$, and for a real vector $a \in \mathbb{R}^{m}$ with positive components, there exists a completely integrable $\bar{P}$ faff system $\left(1_{m n}\right)$ with bounded infinitely differentiable coefficients in $\mathbb{R}_{+}^{m}$ and with lower characteristic set

$$
\Pi\left(A_{1}, \ldots, A_{m}\right)=\left\{p \in \mathbb{R}_{-}^{m}: \alpha_{1} \leq \sum_{i=1}^{m} \frac{p_{i}}{a_{i}} \leq \alpha_{2}\right\}
$$

of positive Lebesgue measure in the space $\mathbb{R}^{m}$.

Proof. 1. The construction of the desired two-dimensional system $\left(1_{m 2}\right)$ and its general solution. Following [4], for the case in which $\alpha_{1}<\alpha_{2}$ we first construct a perfect set $P_{0}$ on $\Delta=[0,1]$ similar to the Cantor perfect set $[10$, p. 50$]$ by using the quantities

$$
\varepsilon_{n}=\exp \left[\left(\alpha_{1}-\alpha_{2}\right) \exp 2^{n+1}\right], \quad n \in \mathbb{N} .
$$

We divide the original closed interval $\Delta_{0}^{(1)}=\Delta$ of zero rank and of length 1 into two closed intervals $\Delta_{1}^{(1)}=\left[0, \varepsilon_{1}\right]$ and $\Delta_{1}^{(2)}=\left[1-\varepsilon_{1}, 1\right]$ of length $\varepsilon_{1}$ of the first rank and one interval $\delta_{1}^{(1)}=\left(\varepsilon_{1}, 1-\varepsilon_{1}\right)$ of the same rank. In a similar way, we split any closed interval $\Delta_{n}^{(m)}, m \in\left\{1, \ldots, 2^{n}\right\}$, of length $\varepsilon_{n}$ and rank $n$ into two closed intervals $\Delta_{n+1}^{(2 m-1)}$ and $\Delta_{n+1}^{(2 m)}$ of length $\varepsilon_{n+1}$ and rank $(n+1)$ whose left and right endpoints, respectively, coincide with those of $\Delta_{n}^{(m)}$, and one interval

$$
\delta_{n+1}^{(m)}=\Delta_{n}^{(m)} \backslash\left(\Delta_{n+1}^{(2 m+1)} \cup \Delta_{n+1}^{(2 m)}\right)
$$

of rank $(n+1)$. We continue this process infinitely; then $\Delta$ contains exactly $2^{n}$ intervals $\Delta_{n}^{(m)}$, $m=1, \ldots, 2^{n}$, and $2^{n-1}$ intervals $\delta_{n}^{(m)}, m=1, \ldots, 2^{n-1}$, for each $n \in \mathbb{N}$.

By $\alpha_{n}^{(m)}, m=1, \ldots, 2^{n}$, we denote the midpoint of $\Delta_{n}^{(m)}$ and introduce the set

$$
P_{0}=\bigcap_{n=1}^{+\infty} \bigcup_{n=1}^{2^{n}} \Delta_{n}^{(m)},
$$

which, by [10, p. 50], has nonzero Lebesgue measure.

On the closed interval $\Delta$, we define the Cantor step function $\Theta_{0}: \Delta \rightarrow[0,1]$ with intervals $\delta_{n}^{(m)}$ of constant values. According to [10, p. 200], this function is a continuous nondecreasing function on $[0,1]$ and has the range $[0,1]=\left\{\Theta_{0}(\alpha): \alpha \in P_{0}\right\}$. Following [4], we introduce the new continuous nondecreasing function

$$
\Theta(\alpha)=\left|\alpha_{2}\right|+\left(\left|\alpha_{1}\right|-\left|\alpha_{2}\right|\right) \Theta_{0}(\alpha):[0,1] \rightarrow\left[\left|\alpha_{2}\right|,\left|\alpha_{1}\right|\right] .
$$

Throughout the following, to preserve the conventional above-introduced notation of the dimension $m \geq 2$ of the time space $\mathbb{R}_{+}^{m}$ and the superscript $m \in\left\{1, \ldots, 2^{n}\right\}$ on the intervals $\Delta_{n}^{(m)}$ (and their midpoints $\alpha_{n}^{(m)}$ ) and the intervals $\delta_{n}^{(m)}$ of rank $n$ used in the construction of the Cantor perfect set $P_{0} \subset[0,1]$ and to avoid related confusion, in the proof of this theorem, we denote the dimension $m$ of the spaces $\mathbb{R}^{m}$ and $\mathbb{R}_{+}^{m}$ by $m_{0} \geq 2$; i.e., we consider the spaces $\mathbb{R}^{m_{0}}$ and $\mathbb{R}_{+}^{m_{0}}$ and the $m_{0}$-dimensional time $t=\left(t, \ldots, t_{m_{0}}\right) \in \mathbb{R}_{+}^{m_{0}}$.

We split the domain $\mathbb{R}_{+}^{m_{0}}$ of the $m_{0}$-dimensional time $t$ by the planes

$$
\tau(t) \equiv a_{1} t_{1}+\cdots+a_{m_{0}} t_{m_{0}}=e^{n}, \quad n \in \mathbb{N},
$$

into the domains

$$
\left\{t \in \mathbb{R}_{+}^{m_{0}}: e^{n} \leq \tau(t)<e^{n+1}\right\}, \quad n \in \mathbb{N} .
$$


These domains are successively denoted by $\Pi_{n}^{(m)}$ so as to ensure that, for any fixed $n \in \mathbb{N}$, the index $m$ takes all values $1, \ldots, 2^{n}$ and the right boundary of the domain $\Pi_{n}^{(m)}$ coincides with the left boundary of the domain $\Pi_{n}^{(m+1)}$; in addition, the right boundary of the domain $\Pi_{n}^{\left(2^{n}\right)}$ coincides with the left boundary of the domain $\Pi_{n+1}^{(1)}$. In turn, we split any domain $\Pi_{n}^{(m)}$ with left closed boundary $\tau(t)=\tau_{m n}$ into the subdomains

$$
\tilde{\Pi}_{n}^{(m)}=\left\{t \in \Pi_{n}^{(m)}: \tau_{m n} \leq \tau(t)<\tau_{m n} \sqrt{e} \equiv \tau_{m n}^{\prime}\right\}, \quad \tilde{\tilde{\Pi}}_{n}^{(m)}=\Pi_{n}^{(m)} \backslash \tilde{\Pi}_{n}^{(m)} .
$$

In addition, we split the last subdomain $\tilde{\tilde{\Pi}}_{n}^{(m)}$ into the subdomains

$$
\bar{\Pi}_{n}^{(m)}=\left\{t \in \tilde{\tilde{\Pi}}_{n}^{(m)}: \tau_{m n}^{\prime} \leq \tau(t)<\tau_{m n} e^{3 / 4} \equiv \tau_{m n}^{\prime \prime}\right\}, \quad \overline{\bar{\Pi}}_{n}^{(m)}=\tilde{\tilde{\Pi}}_{n}^{(m)} \backslash \bar{\Pi}_{n}^{(m)} .
$$

By straightforward computations, we represent the left boundary $\tau_{m n}$ of the domain $\tilde{\Pi}_{n}^{(m)}$ thus defined as

$$
\tau_{m n}=\exp \left(2^{n}+m-3\right), \quad m=1, \ldots, 2^{n}, \quad n \in \mathbb{N} .
$$

Let us introduce the analog

$$
e_{01}\left(\tau, \tau_{1}, \tau_{2}\right)=\left\{\begin{array}{cll}
\exp \left\{-\ln ^{-2}\left(\tau / \tau_{1}\right) \times \exp \left[-\ln ^{-2}\left(\tau / \tau_{2}\right)\right]\right\} & \text { if } & \tau_{1}<\tau<\tau_{2} \\
i-1 & \text { if } & \tau=\tau_{i}, i=1,2
\end{array}\right.
$$

of the infinitely differentiable Gelbaum-Olmsted function [11, p. 54 of the Russian translation] on the interval $\left[\tau_{1}, \tau_{2}\right]$. By using the function $e_{01}$ and by following [4], we introduce two new functions $f[\tau(t)]$ and $F[\tau(t)], t \in \mathbb{R}_{+}^{m_{0}}$. We introduce a bounded infinitely differentiable function $f[\tau(t)]$ that is equal to $\left|\alpha_{2}\right|$ for $t \in \tilde{\Pi}_{n}^{(m)}$ and for arbitrary $n \in \mathbb{N}$ and $m=1, \ldots, 2^{n}$ and also for $\left\{t \in \mathbb{R}_{+}^{m_{0}}: 0 \leq \tau(t)<1\right\}$. In the domain $\tilde{\tilde{\Pi}}_{n}^{(m)}$, we define this function as follows:

$$
f[\tau(t)]= \begin{cases}\left|\alpha_{2}\right|+\left[\Theta\left(\alpha_{n}^{(m)}\right)-\left|\alpha_{2}\right|\right] e_{01}\left(\tau(t), \tau_{m n}^{\prime}, \tau_{m n}^{\prime \prime}\right) & \text { for } t \in \bar{\Pi}_{n}^{(m)} \\ \Theta\left(\alpha_{n}^{(m)}\right)+\left[\left|\alpha_{2}\right|-\Theta\left(\alpha_{n}^{(m)}\right)\right] e_{01}\left(\tau(t), \tau_{m n}^{\prime \prime}, e \tau_{m n}\right) & \text { for } t \in \overline{\bar{\Pi}}_{n}^{(m)} .\end{cases}
$$

Obviously, this function has the property $f:[0,+\infty) \rightarrow\left[\left|\alpha_{2}\right|,\left|\alpha_{1}\right|\right]$.

We define a bounded infinitely differentiable function $F:[0,+\infty) \rightarrow[0,1]$ by the relations

$$
F[\tau(t)]= \begin{cases}\alpha_{1}^{(1)} & \text { if } 0 \leq \tau(t) \leq 1 \\ \alpha_{n}^{(m)} & \text { if } \tau_{m n}^{\prime} \leq \tau(t)<e \tau_{m n} \\ F\left(\tau_{m n}\right)+\left[F\left(\tau_{m n}^{\prime}\right)-F\left(\tau_{m n}\right)\right] e_{01}\left(\tau(t), \tau_{m n}, \tau_{m n}^{\prime}\right) & \text { if } \tau_{m n}<\tau(t)<\tau_{m n}^{\prime} .\end{cases}
$$

Both functions have bounded partial derivatives of any order with respect to all variables $t_{1}, \ldots, t_{m_{0}}$, which follows from the corresponding properties of the functions $e_{01}\left(\tau(t), \tau_{1}, \tau_{2}\right)$.

By using the functions $E(t), f[\tau(t)]$, and $F[\tau(t)]$, we define the two-dimensional vector function

$$
x(t, c)=\left(c_{1}[E(t)]^{f[\tau(t)]},\left[c_{1} F(\tau(t))+c_{2}\right][E(t)]^{\left|\alpha_{2}\right|}\right) \in \mathbb{R}^{2}, \quad c=\left(c_{1}, c_{2}\right) \in \mathbb{R}^{2}, \quad t \in \mathbb{R}_{+}^{m_{0}},
$$

of an arbitrary constant vector $c \in \mathbb{R}^{2}$ and the $m_{0}$-dimensional time vector $t \in \mathbb{R}_{+}^{m_{0}}$; this function coincides in form with the function $x(t, c)$ in [4] but substantially differs from the latter not only in the number of independent variables $t_{1}, \ldots, t_{m_{0}}$ but also in the definition of the basic functions

$$
E(t), \quad f[\tau(t)], \quad F[\tau(t)] \text { and } \tau(t) .
$$

This function is a general solution of the two-dimensional linear partial differential system

$$
\frac{\partial x}{\partial t_{i}}=A_{i}(t) x, \quad x \in \mathbb{R}^{2}, \quad t \in \mathbb{R}_{+}^{m_{0}}, \quad i=1, \ldots, m_{0},
$$


with bounded infinitely differentiable matrices

$$
A_{i}(t)=\left(\begin{array}{cc}
\frac{\partial f[\tau(t)]}{\partial t_{i}} \ln E(t)+f[\tau(t)] E^{-1}(t) \frac{\partial E(t)}{\partial t_{i}} & 0 \\
\frac{\partial F[\tau(t)]}{\partial t_{i}}[E(t)]^{\left|\alpha_{2}\right|-f[\tau(t)]} & \left|\alpha_{2}\right| E^{-1}(t) \frac{\partial E(t)}{\partial t_{i}}
\end{array}\right), \quad t \in \mathbb{R}_{+}^{m_{0}}, \quad i=1, \ldots, m_{0} .
$$

The proof of the infinite differentiability and boundedness of these matrices in $\mathbb{R}_{+}^{m}$ as well as of the complete integrability of the constructed two-dimensional system $\left(1_{m_{0} 2}\right)$ is similar to the corresponding proofs in [4].

2. Construction of a subset of positive Lebesgue $m_{0}$-measure in the entire lower characteristic set of the two-dimensional linear system $\left(1_{m_{0} 2}\right)$. By the definition of the set $P_{0}$, for any $\alpha \in P_{0}$ and $n \in \mathbb{N}$, there exists an $m=m_{n}(\alpha) \in\left\{1, \ldots, 2^{n}\right\}$ such that

$$
\left|\alpha_{n}^{(m)}-\alpha\right| \leq \frac{\varepsilon_{n}}{2}, \quad m=m_{n}(\alpha), \quad n \in \mathbb{N}
$$

We introduce the concise notation

$$
\tau_{m_{n}(\alpha), n}^{\prime \prime} \equiv \eta_{n}(\alpha) \quad \text { and } \quad \alpha_{n}^{\left(m_{n}(\alpha)\right)} \equiv \alpha_{n}^{m}(\alpha) ;
$$

by $(5), \eta_{n}(\alpha)$ satisfies the estimate

$$
\eta_{n}(\alpha) \leq e \tau_{m_{n}(\alpha), n}(\alpha)<\exp 2^{n+1}, \quad n \in \mathbb{N} .
$$

To estimate $\left|F\left(\eta_{n}(\alpha)\right)-\alpha\right|$ from above, we use the inequality

$$
1 \geq \frac{1}{m_{0}} E(t) \geq \exp \left[-\frac{\tau(t)}{m_{0}}\right], \quad t \in \mathbb{R}_{+}^{m_{0}}
$$

and the inequality $f(\tau(t)) \leq\left|\alpha_{1}\right|$. Inequalities (10) and (11) imply also the inequality

$$
1 \geq\left.\frac{1}{m_{0}} E(t)\right|_{r(t)=\eta_{n}(\alpha)}>\exp \left(-\frac{1}{m_{0}} \exp 2^{n+1}\right)>\exp \left(-\exp 2^{n+1}\right), \quad n \in \mathbb{N} .
$$

Therefore, by the definition of the function $F(\tau(t))$ and the estimate (12), we have the inequalities

$$
\begin{aligned}
2\left|F\left(\eta_{n}(\alpha)\right)-\alpha\right| & =2\left|\alpha_{n}^{(m)}(\alpha)-\alpha\right| \underset{(9)}{\leq} \varepsilon_{n}=e^{\left(\alpha_{1}-\alpha_{2}\right)} \exp ^{n+1} \leq\left[\left.\frac{1}{m_{0}} E(t)\right|_{\tau(t)=\eta_{n}(\alpha)}\right]^{\left|\alpha_{1}\right|-\left|\alpha_{2}\right|} \\
& \leq\left[\left.\frac{1}{m_{0}} E(t)\right|_{\tau(t)=\eta_{n}(\alpha)}\right]^{f\left(\eta_{n}(\alpha)\right)-\left|\alpha_{2}\right|} \leq\left[\left.E(t)\right|_{\tau(t)=\eta_{n}(\alpha)}\right]^{f\left(\eta_{n}(\alpha)\right)-\left|\alpha_{2}\right|},
\end{aligned}
$$

where the penultimate inequality holds by virtue of the left estimate in (11).

By virtue of inequality (13), the considered solution $x(t, a)$ with the initial vector $a=\left(c_{1},-\alpha c_{1}\right)$, $\alpha \in P_{0}, c_{1}=$ const $\neq 0$, satisfies the inequalities

$$
\begin{aligned}
\left.\|x(t, a)\|\right|_{\tau(t)=\eta_{n}(\alpha)} & \leq 2\left|c_{1}\right|\left[\left.E(t)\right|_{\tau(t)=\eta_{n}(\alpha)}\right]^{f\left(\eta_{n}(\alpha)\right.}=2\left|c_{1}\right|\left[\left.E(t)\right|_{\tau(t)=\eta_{n}(\alpha)}\right]^{\Theta\left(\alpha_{n}^{(m)}\right)} \\
m & =m_{n}(\alpha) \in\left\{1, \ldots, 2^{n}\right\}, \quad n \in \mathbb{N}, \quad t \in \mathbb{R}_{+}^{m_{0}}
\end{aligned}
$$

It follows from inequalities (9) that

$$
\alpha_{n}^{\left(m_{n}(\alpha)\right)} \rightarrow \alpha \in P_{0} \subset[0,1], \quad n \rightarrow+\infty .
$$


Therefore, by virtue of the continuity of the function $\Theta(\alpha):[0,1] \rightarrow\left[\left|\alpha_{2}\right|,\left|\alpha_{1}\right|\right]$, we have

$$
\Theta\left(\alpha_{n}^{\left(m_{n}(\alpha)\right)}\right) \rightarrow \Theta(\alpha), \quad \alpha \in P_{0}, \quad n \rightarrow+\infty .
$$

Now let us show that the set $P_{x}$ of lower characteristic vectors of the considered solution $x(t, a)$ contains the set

$$
Q_{\alpha} \equiv\left\{p \in \mathbb{R}_{-}^{m_{0}}: \sum_{i=1}^{m_{0}} \frac{p_{i}}{a_{i}}=-\Theta(\alpha)\right\} .
$$

To this end, in accordance with conditions $\left(3_{1}\right)$ and $\left(3_{2}\right)$, we should prove the relation $l_{x}(p)=0$ and the inequalities $l_{x}\left(p+\varepsilon e_{k}\right)<0, k=1, \ldots, m_{0}$, for any vector $p \in Q_{\alpha}$. Let us obtain the latter inequalities. From (14), we have

$$
\begin{aligned}
l_{x}\left(p+\varepsilon e_{k}\right) & \leq\left.\varliminf_{n \rightarrow \infty} \frac{\Theta\left(\alpha_{n}^{(m)}\right) \ln E(t)-(p, t)-\varepsilon t_{k}}{\|t\|}\right|_{\substack{t \in T_{\alpha} \\
\tau(t)=\eta_{n}(\alpha) \\
n \rightarrow \infty}} \frac{1}{r}\left\{-\left[\Theta\left(\alpha_{n}^{(m)}\right)-\sum_{i=1}^{m_{0}} \frac{p_{i}}{a_{i}}\right]-\frac{\varepsilon}{a_{k}}\right\} \\
& =-\frac{\varepsilon}{r a_{k}}+\left.\lim _{n \rightarrow \infty} \frac{1}{r}\left[\Theta(\alpha)-\Theta\left(\alpha_{n}^{(m)}\right)\right]\right|_{m=m_{n}(\alpha)} \\
& =-\frac{\varepsilon}{r a_{k}}<0, \quad r \equiv\left(\sum_{i=1}^{m_{0}} \frac{1}{a_{i}^{2}}\right)^{1 / 2}, \quad \forall \varepsilon>0, \quad k=1, \ldots, m ;
\end{aligned}
$$

here the last equality in the chain holds by virtue of property (15) and the continuity of the function $\Theta:[0,1] \rightarrow\left[\left|\alpha_{2}\right|,\left|\alpha_{1}\right|\right]$.

To prove property $\left(3_{1}\right)$, let us first justify the inequality $l_{x}(p) \geq 0, p \in Q_{\alpha}$. Let the limit $l_{x}(p)$ be realized along a sequence $\{t(k)\}$ with the property $\|t(k)\| \rightarrow+\infty$ as $k \rightarrow \infty$. Obviously, this sequence has the property $\tau(t(k)) \rightarrow+\infty$ as $k \rightarrow \infty$, and without loss of generality, we can assume that there exist limits

$$
f[\tau(t(k))] \rightarrow f_{0} \in\left[\left|\alpha_{2}\right|,\left|\alpha_{1}\right|\right], \quad F[\tau(t(k))] \rightarrow F_{0} \in[0,1], \quad k \rightarrow \infty .
$$

Again without loss of generality, one can assume that there exists a fixed index $l \in\left\{1, \ldots, m_{0}\right\}$ such that

$$
a_{l} t_{l}(k)=\min _{i=1, \ldots, m_{0}}\left\{a_{i} t_{i}(k)\right\} \quad \forall k \in \mathbb{N} .
$$

[If this condition does not hold for all $k \in \mathbb{N}$ but holds for infinitely many $k \in \mathbb{N}$, then we can rarefy the original sequence $\{t(k)\}$ by deleting the elements for which inequality (16) fails; the successive numbering of terms of the remaining infinite sequence gives a new sequence $\{t(k)\}$ for which condition (16) holds for all $k \in \mathbb{N}$.]

Finally, without loss of generality we assume that only one of the following two possible cases takes place.

1. $t(k) \in \tilde{\Pi}_{n(k)}^{(m(k))}$ for all $k \in \mathbb{N}$.

2. $t(k) \in \tilde{\tilde{\Pi}}_{n(k)}^{(m(k))}$ for all $k \in \mathbb{N}$.

Consider the first case. By using the estimate

$$
\|x(t(k), c)\| \geq\left|c_{1}\right|[E(t(k))]^{\left|\alpha_{2}\right|}, \quad t(k) \in \tilde{\Pi}_{n(k)}^{(m(k))}, \quad k \in \mathbb{N},
$$

for the norm of the solution $x(t, c)$ and similar considerations from the proof of the lemma and by taking into account the inequalities $-p_{i} \geq 0$ and $\Theta(\alpha) \geq\left|\alpha_{2}\right|$, we obtain the estimates

$$
\begin{aligned}
l_{x}(p) & \geq \varliminf_{k \rightarrow \infty} \frac{\left|\alpha_{2}\right| \ln E(t(k))-(p, t(k))}{\|t(k)\|} \geq \varliminf_{k \rightarrow \infty} \frac{a_{l} t_{l}(k)\left[-\left|\alpha_{2}\right|-S(p)\right]}{\|t(k)\|} \\
& \geq \varliminf_{k \rightarrow \infty} \frac{a_{l} t_{l}(k)\left[\Theta(\alpha)-\left|\alpha_{2}\right|\right]}{\|t(k)\|} \geq 0, \quad p \in Q_{\alpha} .
\end{aligned}
$$


Now consider the second case. In this case, by the construction of the function $F[\tau(t)]$, for $F_{0}=\alpha$, we have the relations

$$
F[\tau(t(k))]=\alpha_{n(k)}^{(m(k))} \rightarrow \alpha, \quad k \rightarrow \infty .
$$

The construction of the function $f[\tau(t)]$ implies the inequalities

$$
\left|\alpha_{2}\right| \leq f[\tau(t(k))] \leq \Theta\left(\alpha_{n(k)}^{(m(k))}\right), \quad t(k) \in \tilde{\tilde{\Pi}}_{n(k)}^{(m(k))}, \quad k \in \mathbb{N} .
$$

Therefore, by virtue of the inequalities

$$
0<\frac{1}{m_{0}} E(t) \leq 1, \quad t \in \mathbb{R}_{+}^{m_{0}}
$$

and inequality (20), the norm of the solution $x(t, c)$ can be estimated as

$$
\|x(t(k), c)\| \geq\left|c_{1}\right|[E(t(k))]^{f[\tau(t(k))]} \geq\left|c_{1}\right| m_{0}^{\left|\alpha_{2}\right|-\left|\alpha_{1}\right|}[E(t(k))]^{\Theta\left(\alpha_{n(k)}^{(m(k))}\right)}, \quad t(k) \in \tilde{\tilde{\Pi}}_{n(k)}^{(m(k))}, \quad k \in \mathbb{N} .
$$

This, together with the inequalities $p_{i} \geq 0$, condition (16), and the limit relation (19), provides the desired inequalities

$$
\begin{aligned}
l_{x}(p) & \geq \varliminf_{k \rightarrow \infty} \frac{\Theta\left(\alpha_{n(k)}^{(m(k))}\right) \ln E(t(k))-(p, t(k))}{\|t(k)\|} \geq \varliminf_{k \rightarrow \infty} \frac{-a_{l} t_{l}(k)\left[\Theta\left(\alpha_{n(k)}^{(m(k))}\right)+S(p)\right]}{\|t(k)\|} \\
& \geq \varliminf_{k \rightarrow \infty}\left[-a_{l}\left|\Theta\left(\alpha_{n(k)}^{(m(k))}\right)-\Theta(\alpha)\right|\right]=0, \quad p \in Q_{\alpha} .
\end{aligned}
$$

It remains to consider the subcase $F_{0} \neq \alpha$ of the second case. Then, for sufficiently large $k$, the norm of the solution $x(t, c)$ satisfies the estimate

$$
\|x(t(k), c)\| \geq \frac{1}{2}\left|c_{1}\right|\left|F_{0}-\alpha\right|[E(t(k))]^{\left|\alpha_{2}\right|}, \quad t(k) \in \tilde{\tilde{\Pi}}_{n(k)}^{(m(k))}, \quad k_{0} \leq k \in \mathbb{N},
$$

similar to the estimate (17) in the first case. By using this estimate and by performing considerations similar to the proof of the estimate (18), we obtain the desired inequality $l_{x}(p) \geq 0$ in this subcase as well.

Thus, the inequality $l_{x}(p) \geq 0$ has been proved for all $p \in Q_{\alpha}$. To prove the desired relation $l_{x}(p)=0$ in property $\left(3_{1}\right)$, we suppose the contrary: there exists a $p \in Q_{\alpha}$ such that $l_{x}(p)>0$. Then we arrive at a contradiction in the following way:

$$
\begin{aligned}
l_{x}\left(p+\varepsilon e_{i}\right) & =\varliminf_{t \rightarrow+\infty} \frac{\ln \|x(t)\|-(p, t)-\varepsilon_{i} t_{i}}{\|t\|} \geq \underset{t \rightarrow+\infty}{\varliminf_{t \rightarrow+\infty}} \frac{\ln \|x(t)\|-(p, t)}{\|t\|}+\varliminf_{t \rightarrow+\infty}\left(-\varepsilon_{i} \frac{t_{i}}{\|t\|}\right) \\
& =l_{x}(p)-\varepsilon_{i}>0 \quad \forall i=1, \ldots, m_{0},
\end{aligned}
$$

for all $\varepsilon_{i} \in\left(0, l_{x}(p) / 2\right)$.

We have thereby proved the inclusion

$$
Q_{\alpha} \subset P_{x(\cdot, \alpha)}, \quad a=\left(c_{1},-\alpha c_{1}\right) \neq 0, \quad \forall \alpha \in P_{0}
$$

and hence the inclusion

$$
Q\left(m_{0}\right) \equiv\left\{p \in \mathbb{R}_{-}^{m_{0}}: \alpha_{1} \leq \sum_{i=1}^{m_{0}} \frac{p_{i}}{a_{i}} \leq \alpha_{2}\right\} \subset \Pi\left(A_{1}, \ldots, A_{m_{0}}\right) .
$$




\section{CONSTRUCTION OF THE ENTIRE CHARACTERISTIC SET \\ OF THE LINEAR SYSTEM $\left(1_{M_{0} 2}\right)$}

Now let us prove the relation

$$
\Pi\left(A_{1}, \ldots, A_{m_{0}}\right)=Q\left(m_{0}\right) .
$$

To this end, we first prove the inequality $p \leq 0$ for any lower characteristic vector

$$
p \in \Pi\left(A_{1}, \ldots, A_{m_{0}}\right) .
$$

Suppose the contrary: there exists a vector $p$ with a component $p_{l}>0$. The vector $p$ is a lower characteristic vector of some nontrivial solution $x(t, c)$ given by relation (6). Since the functions $f[\tau(t)]$ and $F[\tau(t)]$ satisfy the inequalities

$$
\left|\alpha_{2}\right| \leq f[\tau(t)] \leq\left|\alpha_{1}\right|, \quad 0 \leq F[\tau(t)] \leq 1, \quad t \in \mathbb{R}_{+}^{m_{0}},
$$

it follows that the solution $x(t, c)$ is bounded,

$$
\|x(t, c)\| \leq 2\left|c_{1}\right| m_{0}^{\left|\alpha_{1}\right|}+\left|c_{2}\right| m_{0}^{\left|\alpha_{2}\right|}, \quad t \in \mathbb{R}_{+}^{m_{0}} .
$$

Therefore, in the direction $t_{i}=0, i \neq l, t_{l} \rightarrow+\infty$, we have an inequality contradicting condition $\left(3_{1}\right)$ in the definition of a lower characteristic vector $p$. The proof of the inequality $p \leq 0$ is complete.

Now let us prove the second desired inequality

$$
S(p) \geq \alpha_{1} \quad \forall p \in \Pi\left(A_{1}, \ldots, A_{m_{0}}\right) .
$$

We again suppose the contrary: there exists a vector $p \in \Pi\left(A_{1}, \ldots, A_{m_{0}}\right)$ such that $S(p)<\alpha_{1}$. Let $x(t, c)$ be a nontrivial solution (6) for which $p$ is a lower characteristic vector. By virtue of the inequality $E(t) / m_{0} \leq 1$, the norm of this solution admits the estimates

$$
\begin{aligned}
\|x(t, c)\| & \geq\left|c_{1}\right| m_{0}^{\left|\alpha_{2}\right|}\left[E(t) / m_{0}\right]^{f[\tau(t)]} \geq\left|c_{1}\right| m_{0}^{\alpha_{1}-\alpha_{2}}[E(t)]^{\left|\alpha_{1}\right|}, \quad c_{1} \neq 0, \quad t \in \mathbb{R}_{+}^{m_{0}}, \\
\|x(t, c)\| & =\left|c_{2}\right|[E(t)]^{\left|\alpha_{2}\right|} \geq\left|c_{2}\right| m_{0}^{\alpha_{1}-\alpha_{2}}[E(t)]^{\left|\alpha_{1}\right|}, \quad c_{1}=0, \quad c_{2} \neq 0, \quad t \in \mathbb{R}_{+}^{m_{0}} .
\end{aligned}
$$

Some components $p_{i}$ of the vector $p$ can be zero. Without loss of generality, one can assume that the first $s$ of them are negative and all the remaining components are zero,

$$
p_{i}<0, \quad i=1, \ldots, s, \quad s \in\left\{1, \ldots, m_{0}\right\}, \quad p_{s+1}=\cdots=p_{m_{0}}=0 .
$$

We fix some $l \in\{1, \ldots, s\}$ and choose a number $\varepsilon$ satisfying the conditions

$$
0<2 \varepsilon \max _{i, j}\left\{a_{i} / a_{j}\right\}<\alpha_{1}-S(p), \quad 2 \varepsilon<\min _{i=1, \ldots, s}\left\{\left|p_{i}\right|\right\} .
$$

The following cases are possible for an arbitrary vector $t \in \mathbb{R}_{+}^{m_{0}}$ :

$$
\begin{aligned}
\min _{i=1, \ldots, s}\left\{a_{i} t_{i}\right\} & \equiv a_{l} t_{l} \leq \frac{1}{2} \max _{i=1, \ldots, s}\left\{a_{i} t_{i}\right\} \equiv \frac{1}{2} a_{q} t_{q}, \quad l \neq q, \\
a_{l} t_{l} & >\frac{1}{2} a_{q} t_{q} .
\end{aligned}
$$

In case (26), from the estimate (23), relation (24), and the second condition in (25), we have the inequalities

$$
\begin{aligned}
r(t) & \equiv \ln \|x(t, c)\|-(p, t)-\varepsilon t_{s} \geq d+\alpha_{1} a_{l} t_{l}-\sum_{i=1}^{s} \frac{p_{i}}{a_{i}} a_{i} t_{i}-\varepsilon t_{q} \\
& \geq d+a_{l} t_{l}\left[\alpha_{1}-S(p)\right]-\frac{p_{q}}{a_{q}}\left(a_{q} t_{q}-a_{l} t_{l}\right)-\varepsilon t_{q} \\
& \geq d+a_{l} t_{l}\left[\alpha_{1}-S(p)\right]-\left(\frac{1}{2} \frac{p_{q}}{a_{q}}+\frac{\varepsilon}{a_{q}}\right) a_{q} t_{q} \geq d=\mathrm{const}, \quad t \in \mathbb{R}_{+}^{m_{0}} .
\end{aligned}
$$

DIFFERENTIAL EQUATIONS Vol. 45 No. 52009 
In case (27), from the same estimates (23), relation (24), and the first condition in (25), we have the inequalities

$$
r(t) \geq d+a_{l} t_{l}\left[\alpha_{1}-S(p)\right]-\varepsilon t_{q} \geq d+a_{l} t_{l}\left[\alpha_{1}-S(p)-2 \varepsilon a_{l} / a_{q}\right] \geq d=\mathrm{const}, \quad t \in \mathbb{R}_{+}^{m_{0}} .
$$

We have thereby proved the assertion

$$
\alpha_{1}-S(p)>0 \Rightarrow r(t) \geq d=\text { const }, \quad t \in \mathbb{R}_{+}^{m_{0}},
$$

and hence the inequality

$$
l_{x}\left(p+\varepsilon e_{s}\right) \geq 0, \quad \varepsilon>0,
$$

which contradicts condition $\left(3_{2}\right)$ in the definition of a lower characteristic vector $p \in P_{x}$. The proof of the desired inequality (22) is thereby complete.

Finally, let us prove the last desired inequality

$$
S(p) \leq \alpha_{2}, \quad p \in \Pi\left(A_{1}, \ldots, A_{m_{0}}\right) .
$$

Just as above, we suppose the contrary: there exists a vector $p \in \Pi\left(A_{1}, \ldots, A_{m_{0}}\right)$ such that the opposite inequality $S(p)>\alpha_{2}$ holds. The vector $p$ is a lower characteristic vector of some nontrivial solution $x(t, c)$ given by relation (6). This, together with inequalities (21), implies that such a solution can be estimated as

$$
\begin{aligned}
\|x(t, c)\| & \leq\left|c_{1}\right| m_{0}^{\left|\alpha_{1}\right|}\left[E(t) / m_{0}\right]^{\left|\alpha_{2}\right|}+\left(\left|c_{1}\right|+\left|c_{2}\right|\right)[E(t)]^{\left|\alpha_{2}\right|} \\
& \leq\left(2\left|c_{1}\right| m_{0}^{\left|\alpha_{1}\right|}+\left|c_{2}\right|\right)[E(t)]^{\left|\alpha_{2}\right|}, \quad t \in \mathbb{R}_{+}^{m_{0}} .
\end{aligned}
$$

By using them and the first property in $\left(3_{1}\right)$ of the definition of a lower characteristic vector $p$ of a solution $x(t, c)$, we obtain the following contradiction:

$$
0=l_{x}(p) \leq\left.\varliminf_{t \rightarrow \infty} \frac{\left|\alpha_{2}\right| \ln E(t)-(p, t)}{\|t\|}\right|_{t \in T_{a}}=\frac{1}{r}\left[\alpha_{2}-S(p)\right]<0 .
$$

The proof of inequality (28) is complete. We have thereby proved the theorem in the twodimensional case $n=2$.

\section{CONSTRUCTION OF AN $N$-DIMENSIONAL SYSTEM $\left(1_{M_{0} N}\right)$ WITH $M_{0}$-DIMENSIONAL TIME AND WITH LOWER CHARACTERISTIC SET OF POSITIVE LEBESGUE $M_{0}$-MEASURE}

To give a complete proof of the theorem (in the case of an arbitrary $n>2$ ), we supplement the constructed system $\left(1_{m_{0} 2}\right)$ with general solution $x(t, c)$ given by $(6)$ by a completely integrable system such that, in the general solution $z(t, C)$ of the new system $\left(1_{m n}\right)$, the general solution $x(t, c)$ of system $\left(1_{m_{0} 2}\right)$ with a nonzero vector $c \in \mathbb{R}^{2}$ is dominant, and in the case in which $c=0$ and hence $x(t, 0) \equiv 0$, the supplementing system of order $(n-2)$ has a lower characteristic set lying in the lower characteristic set of the two-dimensional system $\left(1_{m_{0} 2}\right)$. By following [4], we choose such a supplementing system in the form

$$
\frac{\partial y}{\partial t_{i}}=\frac{\left|\alpha_{1}\right|}{E(t)} \frac{\partial E(t)}{\partial t_{i}} y, \quad y \in \mathbb{R}^{n-2}, \quad t \in \mathbb{R}_{+}^{m_{0}}, \quad i=1, \ldots, m_{0},
$$

with a new function $E(t)$. Obviously, this system has bounded infinitely differentiable coefficient matrices

$$
B_{i}(t)=\frac{\left|\alpha_{1}\right|}{E(t)} \frac{\partial E(t)}{\partial t_{i}} E_{n-2}, \quad i=1, \ldots, m_{0},
$$

is completely integrable, and has the general solution

$$
y(t, d)=\left(d_{1}, \ldots, d_{n-2}\right)[E(t)]^{\left|\alpha_{1}\right|}, \quad t \in \mathbb{R}_{+}^{m_{0}},
$$


with an arbitrary constant vector $d \in \mathbb{R}^{n-2}$. The same properties hold for the complete system $\left(1_{m n}\right)$ consisting of two block-systems $\left(1_{m_{0} 2}\right)$ and $(29)$, and its general solution $z(t, C)$ has the form

$$
z(t, C)=(x(t, c), y(t, d)) \in \mathbb{R}^{n}, \quad C=(c, d) \in \mathbb{R}^{n}, \quad t \in \mathbb{R}_{+}^{m_{0}} .
$$

This final block-diagonal system has the form

$$
\frac{\partial z}{\partial t_{i}}=\operatorname{diag}\left[A_{i}(t), B_{i}(t)\right] z, \quad z \in \mathbb{R}^{n}, \quad t \in \mathbb{R}_{+}^{m_{0}}, \quad i=1, \ldots, m_{0} .
$$

By the above-proved lemma, any nontrivial solution $y(t, d)$ of system (29) given by (30) has the lower characteristic set $\left\{p \in \mathbb{R}_{-}^{m_{0}}: S(p)=\alpha_{1}\right\}$, which belongs to the lower characteristic set of system $\left(1_{m_{0} 2}\right)$. Therefore, any solution

$$
z(t, C)=(0, y(t, d)) \in \mathbb{R}^{n}
$$

of system (30) corresponding to the vector $C=(0, d) \in \mathbb{R}^{n} \backslash\{0\}$ has the same lower characteristic set. But for the case in which $c \neq 0$, from the representations (6), (30), and (31) and from the estimate

we obtain the inequalities

$$
0<E(t) / m_{0} \leq 1, \quad t \in \mathbb{R}_{+}^{m_{0}}
$$

$$
1 \leq \frac{\|z(t, C)\|}{\|x(t, c)\|} \leq 1+\frac{\|y(t, d)\|}{\|x(t, c)\|} \leq 1+\frac{\|d\|}{\left|c_{1}\right|}[E(t)]^{\left|\alpha_{1}\right|-f[\tau(t)]} \leq 1+\frac{\|d\|}{\left|c_{1}\right|} m_{0}^{\left|\alpha_{1}\right|}, \quad t \in \mathbb{R}_{+}^{m_{0}},
$$

for $c \neq 0$ and

$$
1 \leq \frac{\|z(t, C)\|}{\|x(t, c)\|} \leq 1+\frac{\|d\|}{\left|c_{2}\right|}[E(t)]^{\left|\alpha_{1}\right|-\left|\alpha_{2}\right|} \leq 1+\frac{\|d\|}{\left|c_{2}\right|} m_{0}^{\left|\alpha_{1}\right|}, \quad t \in \mathbb{R}_{+}^{m_{0}},
$$

for $c_{1}=0$ and $c_{2} \neq 0$. These inequalities can be represented by the single inequality

$$
1 \leq \frac{\|z(t, C)\|}{\|x(t, c)\|} \leq 1+\frac{\|d\| m_{0}^{\left|\alpha_{1}\right|}}{\left|c_{1}\right|+\left|c_{2}\right|\left(1-\operatorname{sgn}\left|c_{1}\right|\right)}, \quad t \in \mathbb{R}_{+}^{m_{0}},
$$

which implies the equivalence of norms of the solutions $z(t, C)$ and $x(t, c)$ for the case in which $c \neq 0$.

Therefore, the lower characteristic set of the $n$-dimensional system (32) with $m_{0}$-dimensional time coincides with the lower characteristic set of the two-dimensional system $\left(1_{m_{0} 2}\right)$ with time of the same arbitrary dimension $m_{0} \geq 2$.

For $\alpha_{1}=\alpha_{2}=\alpha \leq 0$, the desired system $\left(1_{m_{0} n}\right)$ has a form similar to the corresponding system in [4], and by a lemma in the present paper, its lower characteristic set coincides with the set $\left\{p \in \mathbb{R}_{-}^{m}: S(p)=\alpha\right\}$. The proof of the theorem is complete.

\section{REFERENCES}

1. Gaishun, I.V., Vpolne razreshimye mnogomernye differentsial'nye uravneniya (Completely Integrable Multidimensional Differential Equations), Minsk: Navuka i Tekhnika, 1983.

2. Gaishun, I.V., Lineinye uravneniya v polnykh proizvodnykh (Linear Total Differential Equations), Minsk: Navuka i Tekhnika, 1989.

3. Grudo, E.I., Characteristic Vectors and Sets of Functions of Two Variables and Their Fundamental Properties, Differ. Uravn., 1976, vol. 12, no. 12, pp. 2115-2128.

4. Izobov, N.A., On the Existence of Linear Pfaffian Systems Whose Set of Lower Characteristic Vectors Has Positive Plane Measure, Differ. Uravn., 1997, vol. 33, no. 12, pp. 1623-1630.

5. Izobov, N.A., Vvedenie v teoriyu pokazatelei Lyapunova (Introduction to the Theory of Lyapunov Exponents), Minsk: BGU, 2006. 
6. Izobov, N.A., On the Existence of a Linear Pfaff System with Countably Many Distinct Characteristic Sets of Solutions, Differ. Uravn., 1998, vol. 34, no. 6, pp. 735-743.

7. Izobov, N.A., On the Countability of the Number of Solutions of a Two-Dimensional Linear Pfaff System with Distinct Characteristic Sets, Ukrain. Mat. Zh., 2007, vol. 59, no. 2, pp. 172-189.

8. Izobov, N.A., The Set of Lower Exponents of Positive Measure, Differ. Uravn., 1968, vol. 4, no. 6, pp. 1147-1149.

9. Izobov, N.A., Krasovskii, N.G., and Platonov, A.S., The Existence of Linear Pfaff Systems with Lower Characteristic Set of Positive Measure in the Space $R^{3}$, Differ. Uravn., 2008, vol. 44, no. 10, pp. 1311-1318.

10. Natanson, I.P., Teoriya funktsii veshchestvennoi peremennoi (Theory of Functions of a Real Variable), Moscow: Nauka, 1974.

11. Gelbaum, B. and Olmsted, J., Counterexamples in Analysis, San-Francisco: Dover, 1964. Translated under the title Kontrprimery $v$ analize, Moscow: Mir, 1967. 